\title{
Weight Bias During the COVID-19 Pandemic
}

\author{
Rebecca L. Pearl ${ }^{1,2}$ (1) Erica M. Schulte ${ }^{2}$ \\ Accepted: 10 March 2021 / Published online: 18 March 2021 \\ (C) The Author(s), under exclusive licence to Springer Science+Business Media, LLC, part of Springer Nature 2021
}

\begin{abstract}
Purpose of Review This review explores potential sources of weight bias and stigma during the COVID-19 pandemic, including "quarantine-15" messages and discussion of obesity in media and public health campaigns. We examine evidence of the effects of weight bias on well-being during the pandemic and highlight unanswered questions to be addressed in future research.

Recent Findings Studies that have investigated weight change during stay-at-home orders have yielded mixed findings and relied predominantly on self-reported retrospective recall, thus providing weak evidence of a widespread "quarantine-15" effect. No studies to date have evaluated the effects on weight stigma and health of obesity-focused COVID-19 media and public health messages. Individuals with a history of experiencing weight bias may be more vulnerable to binge eating and psychological distress during the pandemic.

Summary Weight bias and stigma during the COVID-19 pandemic, and their effects on health and well-being, warrant greater investigation and consideration in public health efforts.
\end{abstract}

Keywords COVID-19 $\cdot$ Media $\cdot$ Obesity $\cdot$ Quarantine $\cdot$ Weight bias $\cdot$ Weight stigma

\section{Introduction}

The global outbreak of the novel coronavirus disease 2019 (COVID-19) has disrupted everyday life on a massive scale. Concerns for safety and survival remain pressing as the virus continues to spread and cause death, hospitalization, and short- and long-term health impairments that are not yet entirely understood [1-3]. Adding to health concerns is the devastating financial impact the pandemic has had on families [4, 5], as well as inordinate stress for essential workers and for working parents with limited childcare options [6-8]. All of these impacts have been felt most by minority groups and those with the least access to financial and health resources [9-11].

This article is part of the Topical Collection on Psychological Issues

Rebecca L. Pearl

rebecca.pearl@phhp.ufl.edu

1 Department of Clinical and Health Psychology, University of Florida, Gainesville, FL, USA

2 Center for Weight and Eating Disorders, Department of Psychiatry, Perelman School of Medicine at the University of Pennsylvania, Philadelphia, PA, USA
Beyond the direct effects of the novel coronavirus, questions have been raised about the broader impact of the pandemic and its resulting hardships on health and well-being. Speculation about the effects of stress, disrupted routines, and stay-at-home (or "quarantine") orders on health behaviors - such as eating habits, physical activity, and sedentary behavior-has been a prominent topic among health professionals [12-14]. Some obesity researchers and practitioners have predicted that these changes may lead to weight gain in youth and adults $[12,14,15]$. Apprehension about weight gain has been echoed by the general public, as demonstrated across social media platforms with the trending topic of "quarantine-15" (a riff on the term "freshman-15") [16]. In addition, widespread media coverage and public health messages have emerged concerning obesity as a risk factor for heightened risk of severe COVID-19 complications and mortality [17-19]. Together, this media content has been criticized for perpetuating blame toward persons with a higher body weight, promoting unhealthy weight control behaviors, and placing too much emphasis on weight and body image during a deadly and devastating pandemic $[20,21 \bullet, 22 \cdot$. The focus on weight in the context of COVID-19 raises additional questions of how the pandemic may affect weight bias and stigma, which may further increase vulnerability to poorer pandemicrelated outcomes among those targeted. 
In this narrative review, we begin with a brief overview of weight bias and stigma - particularly as they relate to the media, public health messages, and overall health. We then examine three facets of weight bias relevant to the ongoing pandemic: (1) whether discussion of the "quarantine-15" in the media is warranted and/or may contribute to weight bias; (2) why stigma is relevant to public health messages that highlight obesity as a risk factor for heightened COVID-19 severity; and (3) how experiences of weight bias may affect wellbeing during the pandemic. We selected representative peerreviewed studies to include in this review by searching scholarly databases (e.g., PubMed) for articles published in 2020 that included terms such as "COVID-19," "obesity," "eating," "physical activity," "lifestyle change," "weight bias, or "weight stigma." We end by offering a proposed research agenda to deepen knowledge of the contributors to and consequences of weight bias during the COVID-19 pandemic.

\section{Weight Bias and Stigma}

Weight bias refers to negative attitudes toward individuals who are perceived to have excess weight (i.e., overweight or obesity) [23]. Youth and adults with a higher body weight are commonly stereotyped as lazy, lacking self-control, sloppy, unintelligent, and unattractive, among other derogatory connotations [24]. While other forms of implicit bias have reduced in recent years, implicit weight bias continues to increase $[25 \bullet \bullet$. Persons with obesity are also the targets of explicit expressions of weight bias from family, friends, educators, coworkers, peers, and strangers [26, 27]. Weight bias is present in institutional policies, such as the lack of legal protections against weight-based discrimination and bullying [28]. Some individuals also internalize the pervasive societal messages of devaluation due to weight and apply negative stereotypes to themselves, known as weight bias internalization or weight self-stigma [29, 30]. Weight bias may become internalized after experiencing stigma firsthand [31, 32], or through absorbing negative messages about weight from the broader sociocultural environment, including the media.

\section{Weight Bias in Media}

Stigmatizing media portrayals of persons with obesity are common and known to perpetuate weight-biased attitudes $[33,34]$. Across all forms of popular, news, and children's media, individuals with a higher weight are depicted in unflattering images (such as with close-ups of body parts and illfitting clothing) and shown engaging in stereotypical unhealthy eating or sedentary behavior, contributing to the popular notions that these individuals are lazy and slovenly [35-37]. Social media content in forums such as Facebook, Twitter, and Instagram include memes that mock or disparage persons with a higher weight, along with "thin ideal" images that are criticized for promoting unhealthy body image and encouraging extreme dieting behaviors [38-41].

Public health messages and campaigns may also contribute to weight bias and blame toward persons with a higher weight [42]. In experimental studies, health communications that use a negative tone toward persons with obesity, or those that highlight the importance of "personal responsibility" and individual health behaviors, are perceived as stigmatizing and rated as less motivating than are more neutral or positive campaigns that promote environmental changes and healthy behaviors for all, regardless of weight [43-45]. Discussion in news media of the health risks associated with obesity and resulting health care costs may also contribute to derogatory and blaming attitudes toward people who are viewed as burdening the health care system due to their weight $[46,47]$.

\section{Weight Bias and Health}

The experience of weight bias - which may include weightbased teasing, bullying, discrimination, unfair treatment, or subtle microaggressions such as being treated with less courtesy or respect than others - is robustly associated with adverse mental and physical health outcomes [48••]. People who report experiencing weight bias, compared to those who do not, are at greater risk for depression, anxiety, substance use, eating disorders, and low body- and self-esteem [49]. These individuals also report greater overall stress, which may explain links between experiencing weight bias and heightened risk for cardiometabolic risk factors such as dysregulated cortisol and inflammation [50]. All of these associations are significant above and beyond the effects of body weight and other related factors that could influence health, highlighting the unique contribution of stigma to health detriments [51].

Individuals who internalize weight bias (or self-stigmatize) similarly report impaired mental and physical health [52]. Internalization of stigma is associated with reduced selfefficacy to engage in healthy eating and physical activity $[32,53]$. Both experiences and internalization of weight bias have been associated with weight gain and reduced weight loss over time [54-58], although research in this area is relatively new and has produced mixed findings [55, 59, 60]. Altogether there is a consensus in the literature that rather than promoting health behaviors and weight loss, weight bias has an adverse impact on overall health and well-being [48••].

Weight bias also emerges in health care settings, both among health care professionals and in institutional policies [48, 52, 61]. Negative attitudes toward patients with obesity held by health care professionals can impair provider-patient communication and the quality of care provided, while also leading patients to avoid health care due to disrespectful treatment [61]. Body mass index (BMI) cutoffs are frequently used 
to discriminate against patients with high BMIs for certain treatments, including orthopedic surgeries, fertilities treatments, and even bariatric surgery [62-65]. Patients with obesity who are denied care because of their weight are put at greater risk of health decline and exacerbation of current symptoms or disease, contributing to poorer health and quality of life [61]. Thus, through interpersonal experiences of mistreatment due to weight, internalization of negative societal weight messages (e.g., from the media), and discrimination in health care (among other institutional settings), weight bias undermines the mental and physical health of those targeted.

\section{Examination of the "Quarantine-15"}

Terms such as "quarantine-15" and "covid-15" have recently appeared in media to allude to the experience or fear of weight gain as a result of COVID-19 stay-at home orders [16]. For example, social media posts tagged with these terms commonly include stigmatizing "before-and-after quarantine" images that reveal exaggerated weight gain or stereotypical pictures of individuals with a higher body weight eating large amounts of food [16]. News stories covering the "quarantine-15" suggest the inevitability of weight gain during this time period while simultaneously emphasizing the importance of diet and exercise to stay fit [21•]. Contrary to these widespread messages, limited empirical support has been found for the existence of large, population-level weight gain as a result of COVID-19 restrictions, indicating that "quarantine-15" warnings are likely overstated.

\section{Weight Change}

Emerging studies on this topic have found significant variability in reported changes in weight over the course of the pandemic. For example, several large-scale, cross-sectional surveys conducted in the US, India, and Italy have asked participants to retrospectively self-report, at one time point, whether their weight changed (gained, maintained, or lost weight) during quarantine (e.g., spring or summer 2020) compared to before lockdown orders went into effect in March 2020 [66, 67]. In these studies, $19.5-31.5 \%$ of participants reported weight gain, 47.9-59.0\% reported maintaining their weight, and $13.8-19.0 \%$ of individuals reported weight loss [66-68]. While a substantial percentage of participants in these studies did report weight gain, more participants reported no change in their weight, and a significant minority reported weight loss. In one of the few prospective studies conducted to date, no significant changes in self-reported weight were observed in a subsample of 853 adults enrolled in the NutriQuebec cohort study, assessed between June 2019 and February 2020 and again in April or May 2020 [69••].
Another prospective study of weight change during the pandemic demonstrated the significant bias introduced by retrospective recall measures $[70 \bullet \cdot$. Ninety US undergraduate students completed an online survey in January (before the COVID-19 crisis in the US) and again in April 2020. In both surveys, participants self-reported height and weight (to compute BMI) and, in April, also rated on a Likert scale how much they perceived that their weight had changed since the pandemic began (as well as changes in their concerns about weight and shape). Results showed that $28.4 \%$ of participants reported that they had gained weight, and $65.9 \%$ reported that they became more concerned about their weight and shape. In contrast, no significant changes were found in the raw values of self-reported weight and BMI between January and April $[70 \bullet \cdot$. These findings highlight the limitations of surveys that rely on "perceived" changes and that ask individuals to reflect on their pre-pandemic weight. This study assessed young adults; knowledge of the effects of the pandemic on weight is further narrowed by the lack of studies assessing weight change in children and adolescents.

In addition, little is known about how the pandemic has affected weight among individuals actively engaged in weight management. In a study of 150 Italian adults with obesity who were enrolled in a weight-loss treatment program prior to the start of the pandemic, participants completed a questionnaire in April 2020 in which they reported their current weight and their weight from one month prior in March (which they had recorded as part of the treatment program) [71•]. Results showed an average reported weight gain of $1.5 \mathrm{~kg}$. Though notable, this weight change was far less than projected in "quarantine-15" messages, and there was significant individual variability (weight change ranged from -7 to $+10 \mathrm{~kg}$ ) [71•]. Another retrospective, multinational online study of 7753 adults found that individuals with obesity (defined based on BMI) were more likely than those with lower BMIs to report weight gain during the lockdown $(33.4 \%$ vs. $20.5 \%$ of persons categorized as overweight and $24.7 \%$ of those categorized as "normal" weight) [72]. Thus, individuals with obesity may be more prone to experiencing (or perceiving) weight gain during the pandemic. Findings from a crosssectional online study of 2002 adults living in the United Kingdom (UK) surveyed between April and May 2020 suggested that self-efficacy (or confidence) to engage in weight management behaviors was particularly reduced for individuals with a BMI of $35 \mathrm{~kg} / \mathrm{m}^{2}$ or above [73]. However, it is not clear whether these perceptions translate to actual changes in physical activity and eating habits.

\section{Changes in Health Behaviors}

Across studies of adults, adolescents, and children in North America, Europe, and Asia, the most consistent findings include reported decreases in physical activity and increases in 
screen time (e.g., watching television, using the Internet) [66-68, 73-77]. For example, a study in Spain surveyed 516 parents about the behaviors of 860 children and adolescents in April 2020 (retrospectively recalling their children's behavior prior to mandated confinement) and again in May 2020 (recalling behaviors during confinement) [77]. Results showed a significant average reduction in physical activity of more than 100 min per week and a significant average increase in screen time of $2.9 \mathrm{~h}$ per day. Standard deviations were high (160 min of physical activity and $2.1 \mathrm{~h}$ of screen time), indicating significant variability in the sample.

Reported changes in eating behaviors appear to be even more variable [69, 73-77]. In a cross-sectional, online survey in which 723 UK adults retrospectively compared their eating habits in late April 2020 to their typical patterns before the start of quarantine in March 2020,32\% of adults reported eating less healthy during quarantine and $30 \%$ reported eating healthier [75]. Mixed findings have also been observed for specific changes in the types of foods consumed (e.g., ultraprocessed snacks and fast food) [74]. The aforementioned NutriQuebec cohort study compared 24-h food recalls completed in April or May 2020 to those completed before the COVID-19 pandemic (between June 2019 and February 2020) [69••]. On average, adults reported small but significant improvements on the Healthy Eating Index, including increased intake of whole grains, vegetables, and protein and decreased consumption of refined grains and added sugar [69••]. Improvements were greater among individuals who were younger, had lower education, or had a BMI of $30 \mathrm{~kg} /$ $\mathrm{m}^{2}$ or greater $[69 \cdot \bullet]$. However, the authors note that sodium intake increased, and this study collected data relatively early in the national lockdown, precluding conclusions about longer term dietary changes.

Altogether, the evidence suggests that comparable percentages of adults have reported reductions and increases in unhealthy eating in the immediate aftermath of COVID-19 lockdown restrictions, with the only prospective study suggesting small improvements in healthy eating overall and particularly for individuals with obesity [69••]. Few studies have assessed eating behavior changes in youth [74, 76, 77], although some evidence suggests increased consumption of snack foods and sugary beverages and reduced consumption of fruits and vegetables during the pandemic [76, 77]. Questions have been raised about the possible adverse effects of COVID-19 school closures on youth nutrition and weight [15], for which longterm data are needed.

\section{Summary of the Evidence}

Few studies to date have assessed changes in weight over the course of the COVID-19 pandemic. The majority of available data on weight and health behaviors relies on retrospective recall and self-report, which are prone to significant bias. Although some individuals certainly have experienced weight gain during this stressful time, and longer term effects remain unknown, the overall mixed findings do not support the existence of a widespread "quarantine-15" phenomenon. Variability in survey responses is likely due to the myriad of factors that may affect health behaviors during the pandemic, not limited to employment and job sector, food insecurity, and overall stress [78, 79]. The impact of stay-at-home orders and disrupted routines on health warrants further attention, particularly in potentially vulnerable groups such as youths and individuals who are actively trying to manage their weight. Research is also needed to explore the impact of "quarantine-15" messages and other weight-related media content on weight bias, health behaviors, weight, and overall well-being.

\section{Coverage of Obesity and Risk of COVID-19 Complications}

Obesity (particularly class III) has been identified as a risk factor for heightened severity of COVID-19 symptoms and mortality [17, 80-82]. Proposed mechanisms that may explain these associations include low-grade inflammation and impaired immune and respiratory functioning-all of which have heightened prevalence in persons with obesity and can worsen the trajectory of the virus $[83,84,85 \bullet]$. Still, there remain gaps and limitations in the scientific understanding of the link between obesity and COVID-19 outcomes, such as whether these associations account for race/ethnicity, socioeconomic status, or other social determinants of health [86].

It is important to take seriously emerging associations between obesity and COVID-19 risk factors. However, media coverage and public health campaigns aimed at raising awareness of these associations have been criticized for conveying messages of stigma and blame $[21 \bullet, 22 \bullet, 87]$. For instance, news stories have emphasized the need for individual responsibility to reduce the risk of COVID-19 by losing weight through diet and exercise and have included stereotypical images of persons with a higher weight [21•]. The most notable example of the "personal responsibility" narrative from a public heath campaign comes from the UK National Health Service's (NHS) Better Health initiative, launched in July 2020 in response to the pandemic. The official policy document includes statements such as "Tackling obesity would reduce pressure on doctors and nurses in the NHS, and free up their time to treat other sick and vulnerable patients," and "Going into this winter, you can play your part to protect the NHS and save lives" [88]. These messages suggest that those who have a higher weight are burdening health care professionals and the health care system, as well as putting lives at risk. 
Concerns have been raised that such messages of blame and disparagement are counterproductive to efforts to promote health during the pandemic [22•]. As previously noted, weight stigma and approaches that aim to change health behaviors through guilt or shame have been ineffective in the past [89-91]. In addition, the narrative of "personal responsibility" overlooks known biological, socioenvironmental, and psychological factors that play an integral role in determining an individual's body weight [92]. Thus, campaigns that focus predominantly on improving individual health behaviors (such as the UK's recent campaign) do not address key structural barriers that may have a larger impact on population health $[87,92]$.

No studies to date have investigated how messaging in COVID-19 public health campaigns that target individuals with obesity may affect their well-being. Recent research suggests that persons with obesity may be experiencing greater increases in anxiety during the pandemic compared to persons in lower weight categories [72]. It is likely that public discourse around obesity and COVID-19 may be contributing to this distress. Given prior evidence that persons with obesity avoid health care due to stigma [61], attention is due to whether they may avoid COVID-19-related medical care due to concerns about how they will be treated [85•, 93••]. Further, recent discussions have highlighted the potential for weight discrimination if hospitals need to ration care and patients with obesity are deemed less likely to survive COVID-19 (or blamed for their risk factors) [85•]. As such, there is a pressing need for research to evaluate the influence of weight bias and stigma on access to and quality of COVID-19 care, as well as to provide a more nuanced perspective on how to effectively communicate public health initiatives for obesity in a manner that does not perpetuate weight bias and stigma.

\section{Weight Bias and Well-Being During COVID-19}

Few studies to date have directly investigated weight bias in the context of COVID-19. However, existing data suggest that individuals who have experienced weight bias in the past report greater vulnerability to disordered eating and some aspects of psychological distress during the pandemic.

In one study of 584 diverse young adults in Minnesota who were enrolled in a longitudinal cohort study of weight-related health behaviors [94・•], participants who completed surveys in 2018 were re-contacted in the spring of 2020 to complete a survey assessing how COVID-19 was affecting their eating behaviors and well-being. Participants who reported being teased due to their weight prior to the pandemic had 2.4 greater odds of reporting binge eating during the pandemic, even when accounting for pre-pandemic binge eating. Individuals who reported a history of weight teasing also reported greater depression, stress, and eating to cope during the pandemic, although these effects were not significant when accounting for pre-pandemic responses. Weight teasing was not associated with reported physical activity during the pandemic. Altogether, findings from this study suggest that individuals with a history of weight teasing may be uniquely predisposed to binge eating during the pandemic, and their heightened vulnerability to other adverse psychological and behavioral outcomes remains consistent with pre-pandemic levels.

Only two other peer-reviewed publications have directly investigated weight bias in relation to COVID-19. One study surveyed over 2000 US adults in February 2020 (before COVID-19 cases were widespread in the US) about their height, weight, and whether they had ever been treated unfairly (i.e., discriminated against) because of their weight [95••]. Participants were surveyed again in mid-March (when the initial US stay-at-home orders were in effect) about their pandemic-related concerns, preventive behaviors, and changes in their interpersonal relationships. When controlling for demographics, BMI, and whether or not participants were in a "hot zone" for COVID-19 cases, participants who reported weight discrimination (compared to those who did not) reported greater concerns about coronavirus, greater uptake of preventive behaviors, reduced trust in institutions to manage the outbreak, and greater reductions in their relationship quality and feelings of emotional closeness to their community. All effect sizes were small, and no significant differences were found in changes in perceived emotional closeness to family and friends.

A second study followed the same participants at a third assessment in late April 2020 (with a smaller sample of 1590 adults) $[96 \bullet \bullet$. Prior to the pandemic, participants who reported experiencing weight discrimination, compared to those who did not, reported greater depression, anxiety, stress, and loneliness and less life satisfaction, purpose, and social support. These participants also reported greater declines in life satisfaction and purpose and greater incidence of severe depression over the course of the pandemic. Weight discrimination did not predict changes in depression on a continuous scale or changes in loneliness, anxiety, stress, or social support. Raw change scores for all of these variables were relatively small, and ratings of some variables improved. Thus, while weight discrimination was associated with worse baseline psychological well-being on most measures, and these disparities remained consistent, weight discrimination only predicted small differences in trajectories of well-being during the pandemic. Both studies also found no differences in psychological well-being based on BMI alone.

\section{Conclusion}

The purpose of this review was to examine potential sources of weight bias during the COVID-19 pandemic (namely 
"quarantine-15" and other media and public health messages) and to summarize what is known about how weight bias may affect health and well-being under these unique circumstances. Based on a lack of studies, methodological limitations, and mixed findings, we did not find support for a population-level "quarantine-15" phenomenon. News and public health messages about obesity as a risk factor for COVID-19 symptom severity have been criticized for perpetuating weight bias; however, no studies to date have assessed their impact on weight bias and stigma, or on the health care accessed and received by individuals with a higher weight. In addition, while some evidence suggests heightened vulnerability during the pandemic to binge eating and poor mental health among those who have experienced weight bias in the past, no studies to date have assessed experiences or internalization of weight bias and stigma since the start of the pandemic. Thus, there is currently limited knowledge of how COVID-19 and its related restrictions, media coverage, and health care practices may be contributing to existing prejudices, discrimination, or internalization of blame and self-devaluation among individuals with a higher body weight.

Several challenges exist in conducting research during this time. In addition to the aforementioned limitations of retrospective self-report surveys, individuals who are most distressed or under the greatest occupational or financial strain during the pandemic may not be willing to complete survey questionnaires. In the aforementioned study of weight discrimination and health outcomes during the pandemic [96・日, participants who did not complete follow-up assessments were more likely to have endorsed experiencing weight discrimination and reported more depression and loneliness than participants who completed the later assessments. Thus, samples may be biased toward those who have less stigma-related distress and better psychological well-being.

Despite these challenges, several opportunities exist for increasing our understanding of weight bias, and identifying strategies to counteract it, during the COVID-19 pandemic. Figure 1 presents a theoretical model of how weight bias and stigma may affect health and well-being during the pandemic, with specific questions that may be included in a future research agenda. These questions pertain to the prevalence and impact of pandemic-related sources of weight bias (particularly in media and health care) and potential individual factors (such as a history of experiencing weight bias or socioeconomic status) that may moderate or mediate the effects of the pandemic and related weight bias on health. For example, a testable hypothesis is that "quarantine-15" messages might contribute to internalized weight bias, undermine selfefficacy to engage in healthy eating and physical activity, and perpetuate expectations of weight gain throughout

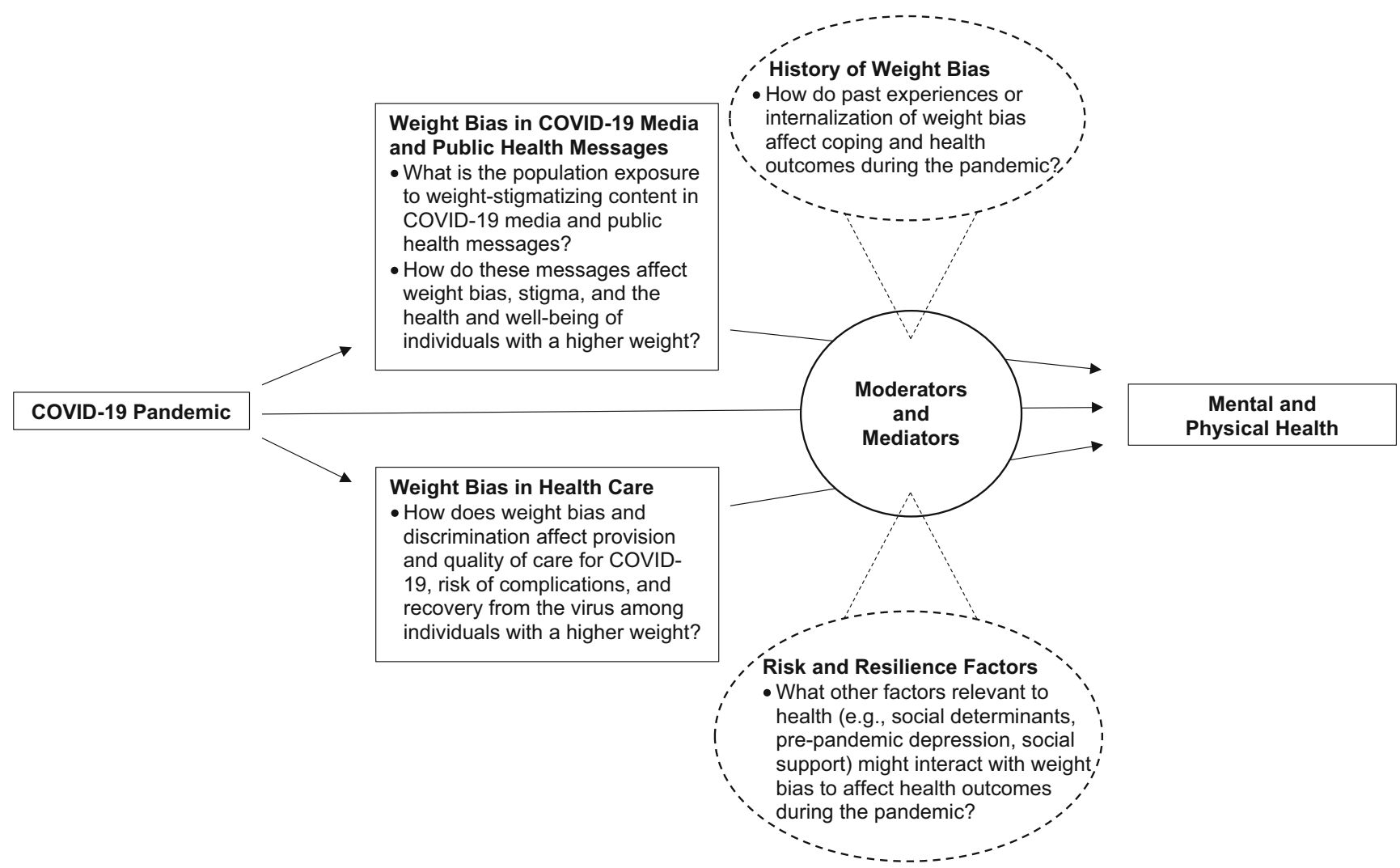

Fig. 1 Theoretical model of proposed research questions to address weight bias during the COVID-19 pandemic 
quarantine [16], which could in turn impact perceived or actual weight gain and, ultimately, health and well-being [96••]. It is also worth examining how news coverage of the links between obesity and COVID-19 severity may contribute to weight bias among the public and health professionals, and the implications this may have for rationing care as COVID-19 hospitalizations rise and resources are strained. Such questions may be investigated through experimental studies (e.g., randomly assigning participants to view different media or public health messages); chart reviews and analyses of hospital data (to investigate potential weight discrimination in COVID-19 treatment); and large-scale surveys of the public, health professionals, and youth and adults with obesity, among other methods. As the pandemic continues, and as weight remains a topic of interest in relation to COVID-19, attention to weight bias and stigma during this time of unprecedented health and safety concerns is critical to preventing additional stress and harm to individuals with a higher body weight.

Funding RLP is supported by a K23 Mentored Patient-Oriented Research Career Development Award from the National Heart, Lung, and Blood Association of the NIH (K23HL140176).

\section{Declarations}

Human and Animal Rights and Informed Consent All reported studies/ experiments with human or animal subjects performed by the authors have been previously published and complied with all applicable ethical standards (including the Helsinki declaration and its amendments, institutional/national research committee standards, and international/national/institutional guidelines).

\section{References}

Papers of particular interest, published recently, have been highlighted as:

- Of importance

•- Of major importance

1. Fraser E. Long term respiratory complications of covid-19. BMJ. 2020;370:m3001.

2. Greenhalgh T, Knight M, Court CA, Buxton M, Husain L. Management of post-acute covid-19 in primary care. BMJ. 2020;370:m3206. https://doi.org/10.1136/bmj.m3026.

3. Weiss P, Murdoch DR. Clinical course and mortality risk of severe COVID-19. Lancet. 2020;395(10229):1014-5.

4. Collins SR, Gunja MZ, Aboulafia GN, Czyzewicz E, Kline CH, Rapoport R et al. An early look at the potential implication of the COVID-19 pandemic for health insurance coverage. 2020. Available from: https://www.commonwealthfund.org/publications/issue-briefs/2020/jun/implications-covid-19-pandemichealth-insurance-survey
5. United States Bureau of Labor Statistics. Supplemental data measuring the effects of the coronavirus (COVID-19) pandemic on the labor market. November 2020. Available from: https://www.bls. gov/covid19/measuring-the-effects-of-the-coronavirus-covid-19pandemic-using-the-current-population-survey.htm

6. Fontanesi L, Marchetti D, Mazza C, Di Giandomenico S, Roma P, Verrocchio MC. The effect of the COVID-19 lockdown on parents: a call to adopt urgent measures. Psychol Trauma. 2020;12(S1): S79-81.

7. Kannampallil TG, Goss CW, Evanoff BA, Strickland JR, McAlister RP, Duncan J. Exposure to COVID-19 patients increases physician trainee stress and burnout. PLOS ONE. 2020;15(8):e0237301. https://doi.org/10.1371/journal.pone. 0237301 .

8. The plight of essential workers during the COVID-19 pandemic. Lancet. 2020;395(10237):1587.

9. Couch KA, Fairlie RW, Xu H. Early evidence of the impacts of COVID-19 on minority unemployment. J Public Econ. 2020;192: 104287.

10. Hooper MW, Napoles AM, Perez-Stable EJ. COVID-19 and racial/ ethnic disparities. Jama. 2020;323(24):2466-7.

11. Rollston R, Galea S. COVID-19 and the social determinants of health. Am J Health Promot. 2020;34(6):687-9.

12. Bhutani S, Cooper JA. COVID-19-related home confinement in adults: weight gain risks and opportunities. Obesity. 2020;28(9): 1576-7.

13. Hall G, Laddu DR, Phillips SA, Lavie CJ, Arena R. A tale of two pandemics: how will COVID-19 and global trends in physical inactivity and sedentary behavior affect one another? Prog Cardiovasc Dis. 2020;64:108-10. https://doi.org/10.1016/j.pcad. 2020.04.005.

14. Mattioli AV, Pinti M, Farinetti A, Nasi M. Obesity risk during collective quarantine for the COVID-19 epidemic. Obes Med. 2020;20:100263. https://doi.org/10.1016/j.obmed.2020.100263.

15. Rundle AG, Park Y, Herbstman JB, Kinsey EW, Wang YC. COVID-19-related school closings and risk of weight gain among children. Obesity. 2020;28(6):1008-9.

16. Pearl RL. Weight stigma and the "quarantine-15". Obesity. 2020;28(7):1180-1.

17. Popkin BM, Du S, Green WD, Beck MA, Algaith T, Herbst CH, et al. Individuals with obesity and COVID-19: a global perspective on the epidemiology and biological relationships. Obes Rev. 2020;21(11):e13128. https://doi.org/10.1111/obr.13128.

18. Public Health England. Major new campaign encourages millions to lose weight and cut COVID-19 risk. July 2020. https://www.gov. uk/government/news/major-new-campaign-encourages-millionsto-lose-weight-and-cut-covid-19-risk.

19. Dastmalchi LN. Battling the "quarantine-15": experts fear COVID19 weight gain could lead to health complications, weak immune systems. ABC News. August 2020. Available from: https:// abcnews.go.com/Health/battling-quarantine-15-experts-fear-covid19-weight/story?id=72572592

20. Your Fat Friend. Your fat friends hear the way you talk about gaining weight during the pandemic. Self. May 2020. Available from: https://www.self.com/story/fat-shaming-pandemic

21. Flint SW. Stigmatizing media portrayal of obesity during the coronavirus (COVID-19) pandemic. Front Psychol. 2020;11:2124. https://doi.org/10.3389/fpsyg.2020.02124 Media coverage during the COVID-19 pandemic has framed obesity as a societal burden and conveyed messages of blame toward people with a higher body weight.

22. The Lancet Diabetes Endocrinology. Obesity and COVID-19: Blame isn't a strategy. Lancet Diabetes Endocrinol. 2020;8(9): 731. https://doi.org/10.1016/S2213-8587(20)30274-6. The recent Public Health England campaign against obesity during the 
COVID-19 pandemic is criticized for using guilt and shame to try to motivate weight loss.

23. Puhl R, Brownell KD. Bias, discrimination, and obesity. Obes Res. 2001;9(12):788-805.

24. Puhl RM, Brownell KD. Psychosocial origins of obesity stigma: toward changing a powerful and pervasive bias. Obes Rev. 2003;4: 213-27.

25.• Charlesworth TES, Banaji MR. Patterns of implicit and explicit attitudes: I. Long-term change and stability from 2007 to 2016. Psychol Sci. 2019;30(2):174-92 While other forms of implicit bias have reduced in the past decade, implicit weight bias has increased.

26. Puhl RM, Brownell KD. Confronting and coping with weight stigma: an investigation of overweight and obese adults. Obesity. 2006;14(10):1802-15.

27. Puhl RM, Lessard LM. Weight stigma in youth: prevalence, consequences, and considerations for clinical practice. Curr Obes Rep. 2020;9:402-11. https://doi.org/10.1007/s13679-020-00408-8.

28. Pearl RL. Weight bias and stigma: public health implications and structural solutions. Soc Issues Policy Rev. 2018;12(1):146-82.

29. Durso LE, Latner JD. Understanding self-directed stigma: development of the Weight Bias Internalization Scale. Obesity. 2008;16: S80-S6.

30. Lillis J, Luoma JB, Levin ME, Hayes SC. Measuring weight selfstigma: the Weight Self-Stigma Questionnaire. Obesity. 2010;18(5):971-6.

31. Pearl RL, Himmelstein MS, Puhl RM, Wadden TA, Wojtanowski AC, Foster GD. Weight bias internalization in a commercial weight management sample: prevalence and correlates. Obes Sci Pract. 2019;5(4):342-53.

32. Pearl RL, Puhl RM, Dovidio JF. Differential effects of weight bias experiences and internalization on exercise among women with overweight and obesity. J Health Psychol. 2015;20(12):1626-32.

33. Ata RN, Thompson JK. Weight bias in the media: a review of recent research. Obes Facts. 2010;3:41-446.

34. Pearl RL, Puhl RM, Brownell KD. Positive media portrayals of obese persons: impact on attitudes and image preferences. Health Psychol. 2012;31(6):821-9.

35. Heuer CA, McClure KJ, Puhl RM. Obesity stigma in online news: a visual content analysis. J Health Commun. 2011;16(9):976-87.

36. Puhl RM, Peterson JL, DePierre JA, Luedicke J. Headless, hungry, and unhealthy: a video content analysis of obese persons portrayed in online news. J Health Commun. 2013;18(6):686-702.

37. Yoo JH, Kim J. Obesity in the news media: a content analysis of obesity videos on YouTube. Health Commun. 2011;27(1):86-97.

38. Boepple L, Ata RN, Rum R, Thompson JK. Strong is the new skinny: a content analysis of fitspiration websites. Body Image. 2016;17:132-5.

39. Chou WS, Prestin A, Kunath S. Obesity in social media: a mixed methods analysis. Transl Behav Med. 2014;4(3):314-23.

40. Lydecker JA, Cotter EW, Palmberg AA, Simpson C, Kwitowski M, White K, et al. Does this Tweet make me look fat? A content analysis of weight stigma on Twitter. Eat Weight Disord. 2016;21:229-35.

41. Wick MR, Harriger JA. A content analysis of thinspiration images and text posts on Tumblr. Body Image. 2018;24:13-6.

42. Stanford FC, Taugeer Z, Kyle TK. Media and its influence on obesity. Curr Obes Rep. 2018;7:186-92.

43. Pearl RL, Lebowitz MS. Beyond personal responsibility: effects of causal attributions for obesity on weight-related beliefs, stigma, and policy support. Psychol Health. 2014;29(10):1176-91.

44. Puhl R, Luedicke J, Peterson JL. Public reactions to obesity-related health campaigns: a randomized controlled trial. Am J Prev Med. 2013;45(1):36-48.
45. Puhl R, Peterson JL, Luedicke J. Fighting obesity or obese persons? Public perceptions of obesity-related health messages. Int $\mathrm{J}$ Obes (Lond). 2013;37(6):774-82.

46. Frederick DA, Saguy AC, Sandhu G, Mann T. Effects of competing news media frames of weight on antifat stigma, beliefs about weight and support for obesity-related public policies. Int J Obes (Lond). 2016;40:543-9.

47. Saguy AC, Frederick D, Gruys K. Reporting risk, producing prejudice: how news reporting on obesity shapes attitudes health risk, policy, and prejudice. Soc Sci Med. 2014;111:125-33.

48.• Rubino F, Puhl RM, Cummings DE, Eckel RH, Ryan DH, Mechanick JI, et al. Joint international consensus statement for ending stigma of obesity. Nat Med. 2020;26:485-97 A joint consensus statement from a modified Delphi process found strong evidence for the prevalence and harms of weight stigma and called for the elimination of weight stigma across health care, media, public health, research, and policy.

49. Papadopoulos S, Brennan L. Correlates of weight stigma in adults with overweight and obesity: a systematic literature review. Obesity. 2015;23:1743-60.

50. Tomiyama AJ. Weight stigma is stressful: a review of evidence for the cyclic obesity/weight-based stigma model. Appetite. 2014;82: $8-15$.

51. Tomiyama AJ, Carr D, Granberg EM, Major B, Robinson E, Sutin AR, et al. How and why weight stigma drives the obesity "epidemic" and harms health. BMC Med. 2018;16(s). https://doi.org/10. 1186/s12916-018-116-5.

52. Pearl RL, Puhl RM. Weight bias internalization and health: a systematic review. Obes Rev. 2018;19(8):1141-63.

53. Pearl RL, Puhl RM, Himmelstein MS, Pinto AM, Foster GD. Weight stigma and weight-related health: associations of selfreport measures among adults in weight management. Ann Behav Med. 2020;54(11):904-14.

54. Jackson SE, Beeken RJ, Wardle J. Perceived weight discrimination and changes in weight, waist circumference, and weight status. Obesity. 2014;22:2485-8.

55. Pearl RL, Wadden TA, Chao AM, Walsh O, Alamuddin N, Berkowitz RI, et al. Weight bias internalization and long-term weight loss in patients with obesity. Ann Behav Med. 2019;53(8):782-7.

56. Puhl RM, Quinn DM, Weisz BM, Suh YJ. The role of stigma in weight loss maintenance among U.S. adults. Ann Behav Med. 2017;51:754-63.

57. Sutin AR, Terracciano A. Perceived weight discrimination and obesity. PLOS ONE. 2013;8(7). https://doi.org/10.1371/journal.pone. 0070048.

58. Wott CB, Carels RA. Overt weight stigma, psychological distress and weight loss treatment outcomes. J Health Psychol. 2010;15(4): 608-14.

59. Lillis J, Thomas JG, Olson K, Wing RR. Weight self-stigma and weight loss during behavioural weight loss intervention. Obes Sci Pract. 2019:5(1):21-7.

60. Olson KL, Lillis J, Thomas JG, Wing RR. Prospective evaluation of internalized weight bias and weight change among successful weight-loss maintainers. Obesity. 2018;26(12):1888-92.

61. Phelan SM, Burgess DJ, Yeazel MW, Hellerstedt WL, Griffin JM, Ryn M. Impact of weight bias and stigma on quality of care and outcomes for patients with obesity. Obes Rev. 2015;16:319-26.

62. Bombak AE, McPhail D, Ward P. Reproducing stigma: interpreting "overweight" and "obese" women's experiences of weight-based discrimination in reproductive healthcare. Soc Sci Med. 2016;166: 94-101.

63. Lui M, Jones CA, Westby MD. Effect of non-surgical, nonpharmacological weight loss interventions in patients who are obese prior to hip and knee arthroplasty surgery: a rapid review. Syst Rev. 2015;4:121. 
64. Tewksbury C, Williams NN, Dumon KR, Sarwer DB. Preoperative medical weight management in bariatric surgery: a review and reconsideration. Obes Surg. 2017;27:208-14.

65. Pillutla V, Maslen H, Savulescu J. Rationing elective surgery for smokers and obese patients: responsibility or prognosis? BMC Med Ethics. 2018;19:28.

66. Scarmozzino F, Visioli F. Covid-19 and the subsequent lockdown modified dietary habits of almost half the population in an Italian sample. Foods. 2020;9(5). https://doi.org/10.3390/foods9050675.

67. Chopra S, Ranjan P, Singh V, Kumar S, Arora M, Hasan MS, et al. Impact of COVID-19 on lifestyle-related behaviours: a crosssectional audit of responses from nine hundred and ninety-five participants from India. Diabetes Metab Syndr. 2020;14(6):2021-30.

68. Zachary Z, Brianna F, Brianna L, Garrett P, Jade W, Alyssa D, et al. Self-quarantine and weight gain related risk factors during the COVID-19 pandemic. Obes Res Clin Pract. 2020;14(3):210-6.

69.• Lamarche B, Brassard D, Lapointe A, Laramée C, Kearney M, Côté $\mathrm{M}$, et al. Changes in diet quality and food security among adults during the COVID-19-related early lockdown: results from NutriQuébec. Am J Clin Nutr. 2021:nqaa363. https://doi.org/10. 1093/ajcn/nqaa363 In a prospective study of adults in the NutriQuebec cohort study, dietary quality improved after the COVID-19 lockdown, particularly among individuals who were younger, had lower education, or had obesity.

70.• Keel PK, Gomez MM, Harris L, Kennedy GA, Ribeiro J, Joiner TE. Gaining "The Quarantine 15:" perceived versus observed weight changes in college students in the wake of COVID-19. Int J Eat. 2020;53(11):1801-8 In a prospective study of weight change related to the COVID-19 lockdown among undergraduate students, weight and BMI did not significantly change over time, but students perceived weight gain and reported increases in weight-related distress.

71. Pellegrini M, Ponzo V, Rosato R, Scumaci E, Goitre I, Benso A, et al. Changes in weight and nutritional habits in adults with obesity during the "lockdown" period caused by the COVID-19 virus emergency. Nutrients. 2020;12(7):2016. https://doi.org/10.3390/ nu12072016 This study of $\mathbf{1 5 0}$ Italian adults with obesity enrolled in a weight loss treatment program observed an average reported weight gain from March to April 2020 of 1. 5 kilograms, with a range from -7 to +10 kilograms.

72. Flanagan EW, Beyl RA, Fearnbach SN, Altazan AD, Martin CK, Redman LM. The impact of COVID-19 stay-at-home orders on health behaviors in adults. Obesity. 2020. Adv Online Publ. 2021. https://doi.org/10.1002/oby.23066.

73. Robinson E, Boyland E, Chisholm A, Harrold J, Maloney NG, Marty L, et al. Obesity, eating behavior and physical activity during COVID-19 lockdown: a study of UK adults. Appetite. 2020;104853:104853. https://doi.org/10.1016/j.appet.2020. 104853.

74. Carroll N, Sadowski A, Laila A, Hruska V, Nixon M, Ma DWL, et al. The impact of COVID-19 on health behavior, stress, financial and food security among middle to high income Canadian families with young children. Nutrients. 2020;12(8):2352.

75. Robinson E, Gillespie S, Jones A. Weight-related lifestyle behaviours and the COVID-19 crisis: an online survey study of UK adults during social lockdown. Obes Sci Pract. 2020. Adv Online Publ. 2020. https://doi.org/10.1002/osp4.442.

76. Pietrobelli A, Pecoraro L, Ferruzzi A, Heo M, Faith M, Zoller T, et al. Effects of COVID-19 lockdown on lifestyle behaviors in children with obesity living in Verona, Italy: a longitudinal study. Obesity. 2020;28(8):1382-5.

77. López-Bueno R, López-Sánchez GF, Casajús JA, Calatayud J, GilSalmerón A, Grabovac I, et al. Health-related behaviors among school-aged children and adolescents during the Spanish Covid19 confinement. Front Pediatr. 2020;8:573. https://doi.org/10. 3389/fped.2020.00573.
78. Sanchis-Gomar F, Lippi G, Lavie CJ. Why is COVID-19 especially impacting the African American population? Ann Med. 2020;52(7):331-3.

79. Cooke JE, Eirich R, Racine N, Madigan S. Prevalence of posttraumatic and general psychological stress during COVID-19: a rapid review and meta-analysis. Psychiatry Res. 2020;292:113347. https://doi.org/10.1016/j.psychres.2020.113347.

80. Cai Q, Chen F, Wang T, Luo F, Liu X, Wu Q, et al. Obesity and COVID-19 severity in a designated hospital in Shenzhen. China. Diabetes Care. 2020;43(7):1392-8.

81. Peng YD, Meng K, Guan HQ, Leng L, Zhu RR, Wang BY, et al. Clinical characteristics and outcomes of 112 cardiovascular disease patients infected by 2019-nCoV. Chinese J Cardiol. 2020;48(6): $450-5$.

82. Lighter J, Phillips M, Hochman S, Sterling S, Johnson D, Francois $\mathrm{F}$, et al. Obesity in patients younger than 60 years is a risk factor for Covid-19 hospital admission. Clin Infect Dis. 2020;71(15):896-7.

83. Gao F, Zheng KI, Wang XB, Sun QF, Pan KH, Wang TY, et al. Obesity is a risk factor for greater COVID-19 severity. Diabetes Care. 2020;43(7):e72-4.

84. Dietz W, Santos-Burgoa C. Obesity and its implications for COVID-19 mortality. Obesity. 2020;28(6):1005-6.

85. Townsend MJ, Kyle TK, Stanford FC. COVID-19 and obesity: exploring biologic vulnerabilities, structural disparities, and weight stigma. Metabolism. 2020;110:154316. https://doi.org/10.1016/j. metabol.2020.154316 Avoidance of health care and weight discrimination in medical settings during the COVID-19 pandemic may exacerbate health disparities for individuals with obesity.

86. Harrison C. Covid-19 does not discriminate by body weight. Wired. April 2020. Available from: https:/www.wired.com/story/covid19-does-not-discriminate-by-body-weight/

87. UK policy targeting obesity during a pandemic - the right approach? Nat Rev Endocrinol. 2020;16(11):609. 10.1038/s41574020-00420-x.

88. Department of Health and Social Care. Tackling obesity: empowering adults and children to live healthier lives. July 2020. Available from: https://www.gov.uk/government/publications/ tackling-obesity-government-strategy/tackling-obesityempowering-adults-and-children-to-live-healthier-lives

89. Duarte C, Matos M, Stubbs RJ, Gale C, Morris L, Gouveia JP, et al. The impact of shame, self-criticism and social rank on eating behaviours in overweight and obese women participating in a weight management programme. PLOS One. 2017;12(1):e0167571. https://doi.org/10.1371/journal.pone.0167571.

90. Mensinger JL, Tylka TL, Calamari ME. Mechanisms underlying weight status and healthcare avoidance in women: a study of weight stigma, body-related shame and guilt, and healthcare stress. Body Image. 2018;25:139-47.

91. Schvey NA, Puhl RM, Brownell KD. The impact of weight stigma on caloric consumption. Obesity. 2011;19(10):1957-62.

92. Brownell KD, Kersh R, Ludwig DS, Post RC, Puhl RM, Schwartz $\mathrm{MB}$, et al. Personal responsibility and obesity: a constructive approach to a controversial issue. Health Aff (Millwood). 2010;29(3): 379-87.

93.• Le Brocq S, Clare K, Bryant M, Roberts K, Tahrani AA. writing group form Obesity UK et al. Obesity and COVID-19: a call for action from people living with obesity. Lancet Diabetes Endocrinol. 2020;8(8):652-4 People living with obesity express fears about contracting COVID-19 and being able to receive proper medical care. They also recognize weight stigma in media coverage of the pandemic.

94.• Puhl RM, Lessard LM, Larson N, Eisenberg ME, Neumark-Sztainer D. Weight stigma as a predictor of distress and maladaptive eating behaviors during COVID-19: longitudinal findings from the EAT Study. Ann Behav Med. 2020;54(10):738-46 Past experience of 
weight teasing was prospectively associated with more binge eating during the COVID-19 pandemic, above and beyond pre-pandemic binge eating. Heightened vulnerability to psychological distress during the pandemic among individuals who had experienced weight teasing remained consistent with pre-pandemic reports.

95.• Sutin AR, Robinson E, Daly M, Gerend MA, Stephan Y, Luchetti $\mathrm{M}$, et al. BMI, weight discrimination, and psychological, behavioral, and interpersonal responses to the coronavirus pandemic. Obesity. 2020;28:1590-4 Pre-pandemic experiences of weight discrimination were associated with greater concerns about COVID-19, greater engagement in preventive behaviors, less trust in institutions, and declines in feelings of social connectedness.
96.• Sutin AR, Stephan Y, Luchetti M, Aschwanden D, Strickhouser JE, Lee $\mathrm{JH}$, et al. Body mass index, weight discrimination, and the trajectory of distress and well-being across the coronavirus pandemic. Obesity. 2020. https://doi.org/10.1002/oby.23048 Individuals with a history of experiencing weight discrimination reported small but significantly greater reductions in some aspects of psychosocial well-being relative to individuals who did not report experiencing weight discrimination.

Publisher's Note Springer Nature remains neutral with regard to jurisdictional claims in published maps and institutional affiliations. 